\title{
Analysis of Void Formation Mechanism in the Vacuum \\ Reflow Soldering Process of Semiconductor Laser Diode
}

\author{
Peidong Xu, Bin Wang, Yong Wang, Xiantao Wang* \\ College of Science, Changchun University of Science and Technology, Changchun 130022, China \\ *wxt@cust.edu.cn
}

\begin{abstract}
In this paper, the vacuum reflow soldering technology for semiconductor laser chips in optoelectronic devices was studied and analyzed in a systematic manner. Through the study on the key elements in the reflow soldering process, such as the selection of solders, chamber vacuum, flux, and the pressure applied by the fixture on the chip, this paper focused on exploring the formation mechanism of voids in the solder layer when the device was resoldered. Also, the change in the movement of gas bubbles in the voids with changing reflow oven chamber conditions and its underlying law were analyzed, by preparing $200 \mathrm{C}$-package semiconductor laser diodes and verifying the reliability and stability of the theoretical analysis through inspection and test aging. which could provide a theoretical basis for the use of the vacuum reflow soldering technology to reduce the void rate in the soldering process of devices.
\end{abstract}

Keywords: Semiconductor laser diode; Voids; Reflow Soldering; Packaging of laser diode; Optoelectronic Devices; Solders

\section{Introduction}

With the rapid development of science and technology, optoelectronic devices have gradually penetrated all walks of life. With high brightness, excellent beam quality, low drive current, direct modulation, and other excellent features, along with the breakthroughs in semiconductor materials and preparation technology, internal material structure improvement, and performance enhancement, semiconductor lasers have been widely used in medical, military, material processing, civil and other fields. For semiconductor lasers, vacuum reflow soldering is one of the important steps in the packaging process. The soldering quality has a direct impact on the output characteristics of a semiconductor laser, even on its lifetime. For example, in the reflow soldering process, due to improper control of temperature, time, pressure, vacuum, mechanical fixation, flux, or other variables, problems such as poor soldering, solder overflow, tilting, high void rate of semiconductor laser chips may occur, which would seriously affect the stability of the device. Owing to it, how to weld devices with high reliability has become a pressing problem. On the basis of the original technology, this paper conducted theoretical research on the escape velocity of gas bubbles in the voids under negative pressure, and analyzed the impact of voids and oxides formed in the soldering process on the soldering quality, with the aim of obtaining semiconductor laser devices with excellent performance.

\section{Key Technologies in Vacuum Reflow Soldering}

By means of the vacuum reflow soldering technology, heating and cooling of the devices can be precisely controlled by relying on its precise temperature control equipment through vacuum reflow ovens and infrared heating. Due to it, the technology has been widely applied in optoelectronic devices, aerospace and military electronics, and other fields. This section focuses on several key technologies in vacuum reflow soldering.

\section{A. Solders}

In the soldering of semiconductor laser chips, solders such as indium (In), Au-Sn alloy (AuSn), SAC305 $(\mathrm{SnAgCu})$ are commonly used. Solders containing gold and indium are easier to combine with gold-plated substrates. For the selection of solders, the melting point of the solder should not be higher than the maximum temperature that the chip can withstand, which shall be taken into consideration first because too high temperature will affect the chip's performance, resulting in failure. Secondly, the thermal conductivity, creep resistance, and yield strength of the solder should be considered. If the coefficient of thermal expansion does not match, the stability of the device will be affected under the effect of thermal stress. Lattice vacancies occur as a consequence of the difference in diffusion rates between two alloys in the diffusion process, resulting in the accumulative formation of more and larger voids. This phenomenon is called Kirkendall effect as 
shown in Figure 1. The Kirkendall effect seriously affects the stability of the device. Therefore, the selection of substrate materials and internal doping elements of solders is of significant importance [1].

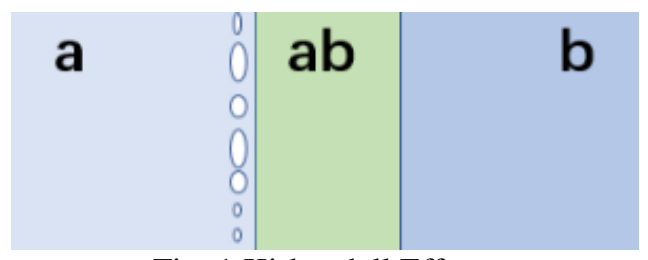

Fig .1 Kirkendall Effect

In addition, the oxide film on the surface of solders is difficult to wet, which affects the stability of the solders and devices. Therefore, the solders need to be surface treated (pickled and plasma cleaned) to remove the surface oxide film before use.

\section{B. Vacuum}

In the vacuum reflow soldering process, by controlling the vacuum inside the chamber, the internal gas or gas bubbles can be discharged to prevent the spattering of solder balls. If the chamber contains oxygen, an oxidation reaction shall take place between oxygen and the solder (M), resulting in the formation of an oxide. The reaction principle can be expressed by the following equation.

$$
2 \alpha \mathrm{M}+\beta \mathrm{O}_{2}=2 \mathrm{M}_{\alpha} \mathrm{O}_{\beta}
$$

The oxide generated on the solder layer is characterized by strong adhesion and can affect solder wetting, resulting in solder voids.

\section{Flux}

Since it is impossible to make a perfect vacuum in the vacuuming process, a certain amount of oxygen will definitely be left in the chamber, making the formation of an oxide inevitable. Therefore, to remove the oxide generated in the reflow soldering process, inert gas $\left(\mathrm{N}_{2}\right)$ or reducing gas (such as $\mathrm{HCOOH}$ and $\mathrm{N}_{2}$ ) can be put into the chamber as the flux to react with the oxide, so as to achieve the purpose of removing the oxide. When the temperature reaches a certain value, a reduction reaction occurs between the formic acid $(\mathrm{HCOOH})$ and the oxide $\left(\mathrm{M}_{\alpha} \mathrm{O}_{\beta}\right)$ with the assistance of nitrogen:

$$
\mathrm{M}_{\alpha} \mathrm{O}_{\beta}+2 \beta \mathrm{HCOOH} \triangleq \mathrm{M}_{\alpha}(\mathrm{HCOO})_{2 \mathrm{y}}+\beta \mathrm{H}_{2} \mathrm{O}
$$

Formate undergoes a decomposition reaction under certain conditions.

$$
\mathrm{M}_{\alpha}(\mathrm{HCOO})_{2 \mathrm{y}} \triangleq \alpha \mathrm{M}+2 \beta \mathrm{CO}+\beta \mathrm{H}_{2}
$$

As we can see, due to the flux, the oxide will be gradually decomposed and disappear with the reduction reaction, and the oxide in the solder layer is reduced, thus causing a decrease in the void rate in the soldering process.

\section{Pressure}

To ensure that the solder is in full contact with the substrate during the soldering process, the wetting of the solder is of particular importance. Therefore, it is necessary to ensure the accuracy of the pressure values during the soldering process. The filler length $\mathrm{L}$ of the solder in soldering can be expressed as

$$
\mathrm{L}=2 \sigma \cdot \cos \theta / \mathrm{h} \cdot \mathrm{G}
$$

where $\sigma$ is the surface tension of the solder, $\theta$ is the contact angle of the solder, $h$ is the gap between the chip and the substrate, and $\mathrm{G}$ is the gravitational acceleration. As we can see from the expression, by applying pressure, $\mathrm{h}$ can be effectively reduced to increase the contact area so as to increase the filler length L. In addition, the value of pressure should be properly controlled. Too large a value will lead to the overflow of solder in the melting process, resulting in a missing solder and increasing void rate $[2,3]$.

\section{Void Formation Mechanism and Solution}

\section{A. Formation Mechanism}

For semiconductor lasers, the soldering quality directly affects their output performance. The voids formed by poor soldering act as an important test parameter. If the void rate is too high, a surge in heat, poor heat dissipation, increased thermal resistance, and spectral broadening of the device shall occur. 
With the passage of time, the soldering area shall be gradually oxidized, which could have a serious impact on the stability and reliability of the device. The schematic diagrams of the voids on the surface of solders are shown in Figure 2 below.

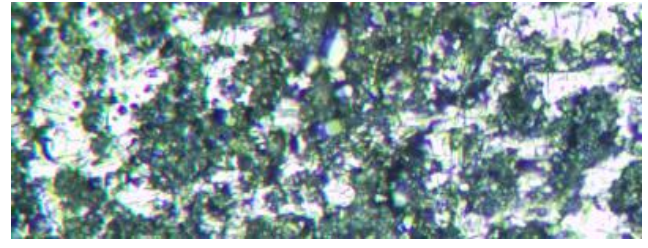

Fig. 2(a) Voids on the surface of

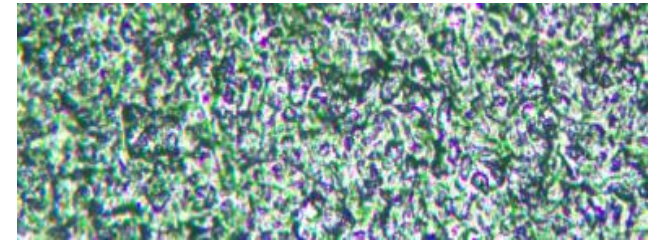

Fig. 2(b) Voids on the surface of the SAC solder

There are many reasons for the formation of voids, which are mainly attributed to the following points.

(1) Preheating temperature is too low. The temperature is lower than the melting point of the solder, resulting in poor wetting and a decrease in bonding area, further causing an incomplete discharge of gas bubbles in the soldering process.

(2) Soldering time is too short and the temperature climbs too quickly, resulting in incomplete melting of the solder and incomplete discharge of gas bubbles.

(3) Substrate surface roughness is not qualified, such as burrs. If there is a certain number of voids, the solder cannot be completely filled.

(4) The oxide film on the solder surface is not completely removed, which will prevent the penetration of the solder, resulting in incomplete wetting of the substrate.

(5) Solder contamination and poor precision of mechanical fixtures occur due to human factors.

\section{B. Solution}

To drive out the bubbles that exist in the voids, a theoretical analysis of the movement trajectory of the bubbles was performed. Firstly, considering the bubble rise velocity, and based on Stokes' theorem, it can be known that the resistance $f$ of the molten solder is

$$
f=3 \pi \cdot \eta \cdot V_{b} \cdot d
$$

where $\eta$ is the solder viscosity, and $V_{b}$ is the free settling velocity of the bubble, and $d$ is the bubble diameters.

When the bubble reaches the equilibrium state, the buoyancy is $F=G+f$. Then the bubble rise velocity $\mathrm{V}_{\mathrm{b}}$ can be expressed as

$$
V_{b}=\frac{2}{9} \cdot \frac{P_{l}-P_{b}}{\eta} \cdot G \cdot r^{2}
$$

where $P_{l}$ is the density of the molten solder, $P_{b}$ is the bubble density, and $G$ is the gravitational acceleration.

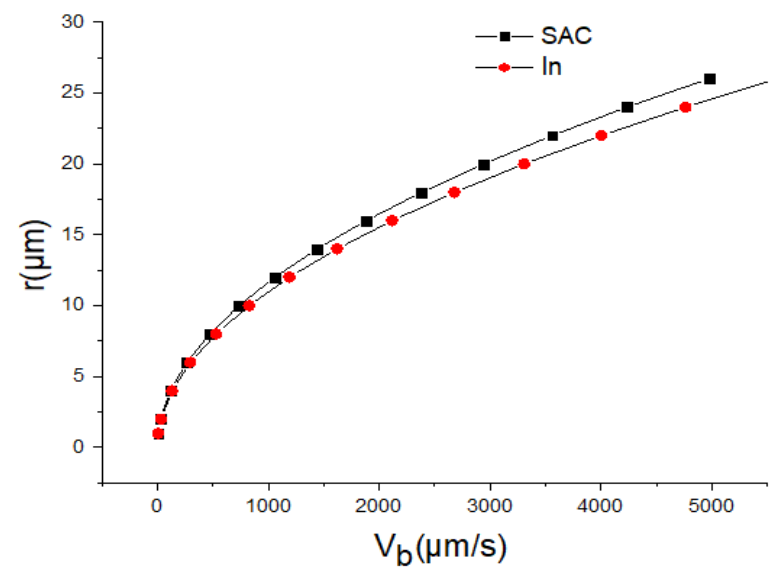

Fig. 3 Relationship between radius and velocity

As shown in Figure 3 above, as the radius of the bubble gradually increases, the bubble rise velocity in the solder layer also increases. According to the formula, a certain rise time is required when the bubble 
in the solder reaches the equilibrium state, so as to ensure complete discharge of the bubbles, resulting in a decrease in the void rate.

Also, according to the equation $\mathrm{p}=\frac{2 \cdot \mathrm{R}_{\mathrm{lv}}}{\mathrm{r}}\left(\mathrm{R}_{\mathrm{lv}}\right.$ is the surface tension of the bubble in the molten solder). Then the relationship between the pressure and the radius of the bubble can be deduced as

$$
p=\sqrt{\frac{8 \cdot R_{l v}^{2} \cdot G \cdot\left(P_{l}-P_{b}\right)}{9 \eta \cdot V_{b}}}
$$

Then the relationship between the pressure in the chamber and the escape velocity of the bubbles can be obtained. It can be seen that the escape velocity of the bubbles will increase with the increase of the pressure. The relationship between the pressure and the movement velocity of the bubbles in the voids is shown in Figure 4 below [4,5].

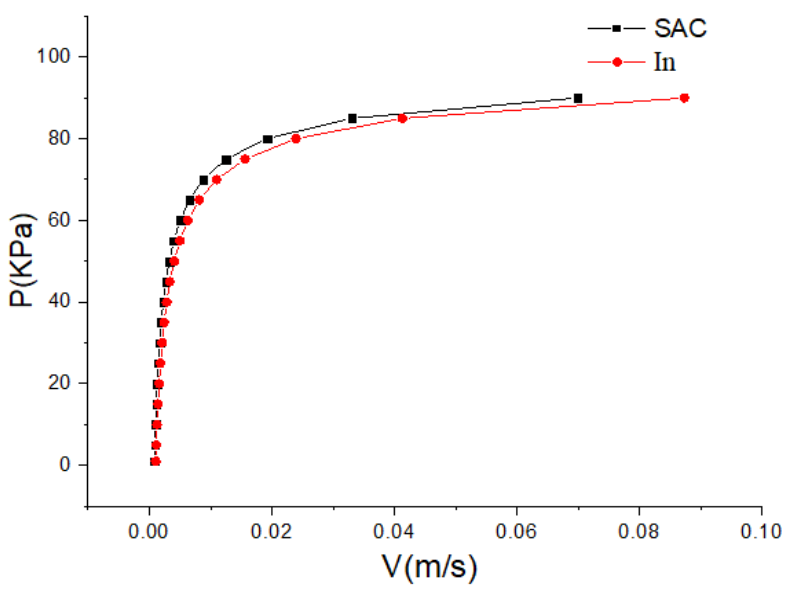

Fig. 4 Relationship between pressure and velocity

In summary, a schematic diagram of the bubble voids formed in a semiconductor laser chip during vacuum reflow soldering is shown in Figure 5 below.

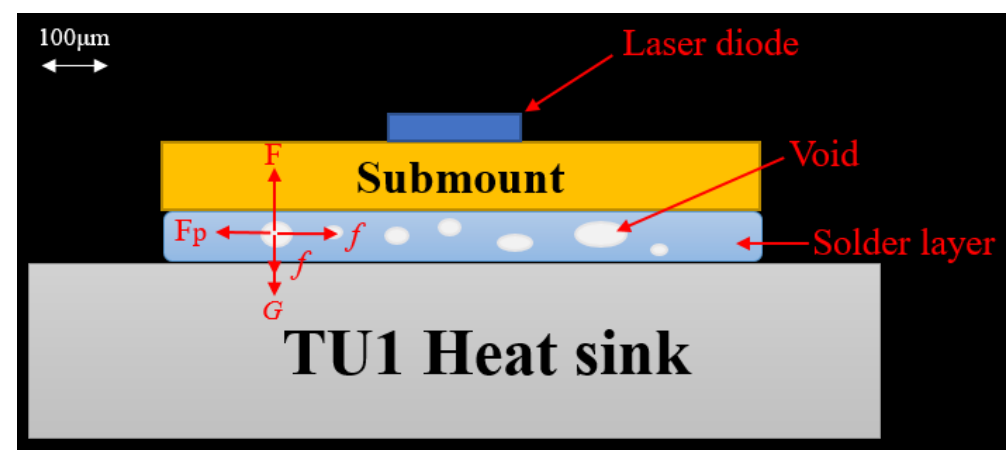

Fig. 5 Schematic diagram of bubble voids

As shown in Figure 5, for soldering COS on an oxygen-free copper substrate, the bubbles in the molten solder layer gradually rise and become stable when a balance is reached among the buoyancy $\mathrm{F}$, the bubble's gravity $\mathrm{G}$, and the viscous resistance $f$. When the chamber is vacuumized, the force exerted by the pressure on the bubble is represented by Fp. Under the action of Fp, the bubble will gradually escape from the solder layer with the increase in pressure so as to reduce the void rate.

If the void rate is to be reduced to improve the soldering strength, the gas inside the chamber needs to be discharged, and the reflow soldering process needs to be carried out in a vacuum environment. Under the condition that the temperature, pressure, and other factors remain constant, the equation of state for ideal gases can be expressed as

$$
\mathrm{PV}=\mathrm{nRT}
$$

It can be simplified as

$$
\mathrm{P}_{\mathrm{a}} \mathrm{V}_{\mathrm{a}}=\mathrm{P}_{\mathrm{b}} \mathrm{V}_{\mathrm{b}}
$$

where $P_{a}$ and $V_{a}, P_{b}$ and $V_{b}$ are the pressure and the volume of the gas inside the chamber before and after soldering, respectively. As can be seen from the formula, when the pressure inside the chamber increases, the corresponding gas volume becomes smaller. In other words, the bubbles in the voids can be gradually discharged, resulting in a decrease in the void rate. It is noteworthy that the ideal 
state cannot be reached by means of current technology and under current conditions, that is, the vacuum cannot be at absolute zero, and the void rate can only be reduced as much as possible but cannot reach zero $[6.7,8]$.

\section{Reliability Verification}

Based on the above theoretical analysis and research on vacuum reflow soldering technology for semiconductor lasers, the group prepared $200 \mathrm{C}$-package structure, wavelength of $1064 \mathrm{~nm}$, output power of $3 \mathrm{~W}$ semiconductor laser to verify the reliability of the device, the following Figure 6 (1) for the preparation of the C-package semiconductor laser physical diagram, Figure 6 (2) for the partial semiconductor laser diode.

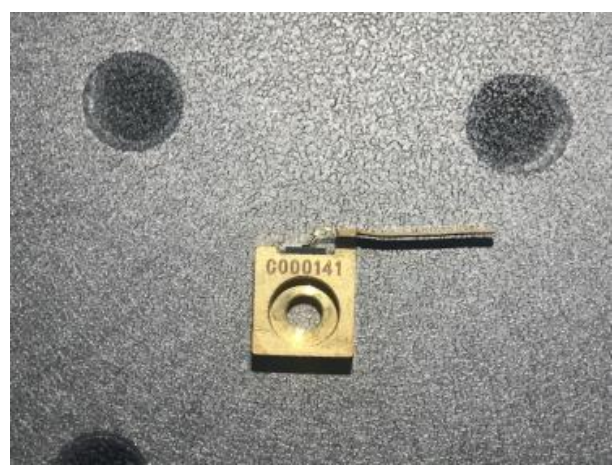

Fig. 6(1) Physical diagram of C-package semiconductor laser diode

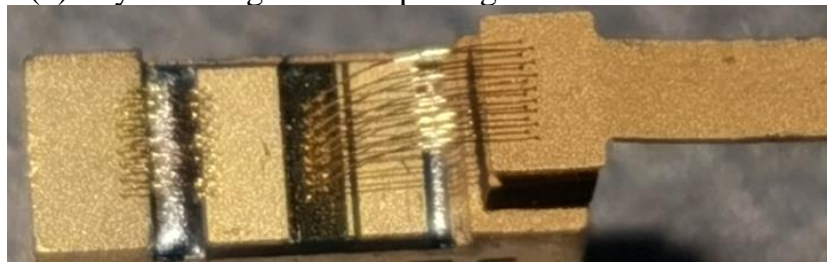

Fig. 6 (2) Partial diagram of C package semiconductor laser diode

The 200 prepared devices were sampled and inspected by the equipment for characterization tests, such as mirror inspection (Figure 7), shear force test, and basic performance parameter tests (P-V-I curve, wavelength, optical power, thermal resistance, etc.), and no defective products were found. Relative to the device before the optimization of vacuum reflow technology, if the device reflow- soldering cavity defects, shear force test sampling process will screen out part of the defective products, in addition to the aging test will also be side characterization of the device with cavity defects [9].

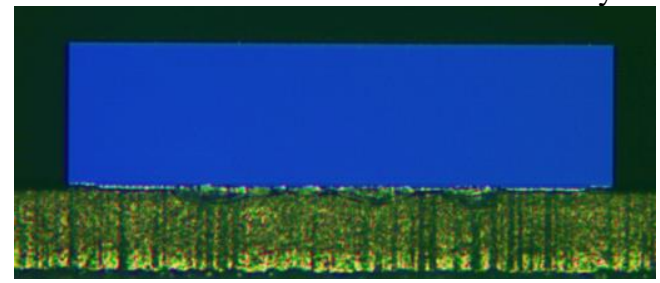

Fig. 7. Physical map of Chip microscopy cavity surface

Finally, after $1500 \mathrm{~h}$ photoelectric performance aging test (Figure 8), draw a conclusion: excluding the chip itself is bad and human operation caused by die-bonding when the solder overflow cause COD, the device qualification rate reached $98 \%$, vacuum reflow process does not appear bad devices, good stability, suitable for mass production. 


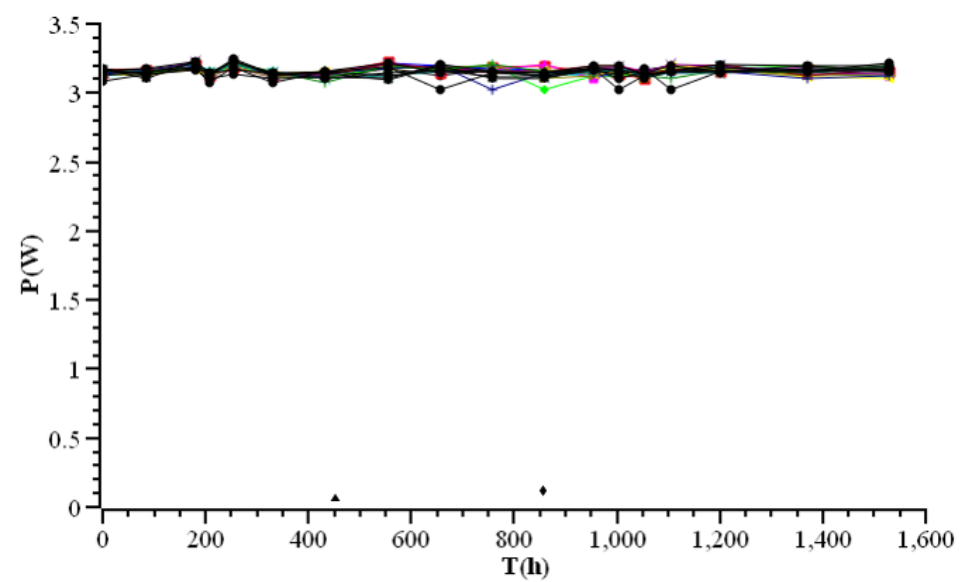

\section{Conclusion}

Fig. 8. The aging test curve of Photoelectric performance

This paper explored the formation mechanism of voids in the vacuum reflow soldering process of semiconductor laser chips in optoelectronic devices and analyzed the impact of solder, vacuum, flux, and pressure on the formation of voids in the reflow soldering process. Through analyzing the relationship between pressure and bubble radius and movement velocity in the reflow chamber, and the movement mechanism of the bubbles in the voids, the impact of external factors in the reflow soldering technology on the internal void bubbles was reflected in terms of change and the law of movement. Finally, the reliability as well as the stability of the theoretical analysis was verified by preparing 200 C-package structure semiconductor lasers, which were tested and aged, which could provide a theoretical basis and reference for the reflow soldering process.

\section{Acknowledgement}

This work was supported by the Jilin Scientific and Technological Development Program (No.20160203017GX).

\section{Disclosures}

The authors declare no conflicts of interest.

\section{References}

[1] Chu H M, Ngoc H V, Hane K. Vacuum package using anodic bonding assisted by the reflow of low-melting temperature metal[J]. International Journal of Precision Engineering \& Manufacturing, 2014, 15(4):695-701.

[2] Wang D, Panton R L. Experimental Study of Void Formation in Eutectic and Lead-Free Solder Bumps of Flip-Chip Assemblies[J]. Journal of Electronic Packaging, 2006, 128(3): p.202-207.

[3] Wang Z D, Luan X H, Zhou Z, Wu FS, Liu H. The two-phase flow simulation and experimental research on the formation of solder voids in power module[J]. Microelectronics Reliability, 109.

[4] P. Wild, D. Lorenz, T. Grözinger, A. Zimmermann. Effect of voids on thermo-mechanical reliability of chip resistor solder joints: Experiment, modelling and simulation[J]. Microelectronics Reliability,2018,85.

[5] Takayama T, Rinderer L. Flux flow velocity and viscosity coefficient of a superconducting indium film[J]. Physica B+c, 1981, 107(1-3):431-432.

[6] Z Xiao，S Liang，Z Pei，T Zhao，B Yu，CG Bao，JF Yang，GJ Qiao. Fabrication of Transparent Alumina by Rapid Vacuum Pressureless Sintering Technology[J]. Journal of the American Ceramic Society, 2012, 95(7):2116-2119. 
[7] Moser Z, Sebo P, Gasior W, Svec P. Effect of indium on wettability of Sn-Ag-Cu solders. Experiment vs. modeling, Part I[J]. Calphad, 2009, 33(1):63-68.

[8] Correlation between viscosity of molten $\mathrm{Cu}-\mathrm{Sn}$ alloys and phase diagram[J]. Physica B Physics of Condensed Matter, 2007, 387(1-2):1-5.

[9] Yan Y, Guan Y, Chen X, GQ Lu. Effects of Voids in Sintered Silver Joint on Thermal and Optoelectronic Performances of High-Power Laser Diode[J]. Journal of Electronic Packaging, 2013, 135(4):041003. 